This item was submitted to Loughborough's Research Repository by the author.

Items in Figshare are protected by copyright, with all rights reserved, unless otherwise indicated.

\title{
Recovery of heart rate variability after treadmill exercise analyzed by lagged Poincaré plot and spectral characteristics
}

PLEASE CITE THE PUBLISHED VERSION

http://dx.doi.org/10.1007/s11517-017-1682-2

\section{PUBLISHER}

(c) International Federation for Medical and Biological Engineering. Published by Springer.

\section{VERSION}

AM (Accepted Manuscript)

\section{PUBLISHER STATEMENT}

This work is made available according to the conditions of the Creative Commons Attribution-NonCommercialNoDerivatives 4.0 International (CC BY-NC-ND 4.0) licence. Full details of this licence are available at: https://creativecommons.org/licenses/by-nc-nd/4.0/

\section{LICENCE}

CC BY-NC-ND 4.0

\section{REPOSITORY RECORD}

Shi, Ping, Sijung Hu, and Hongliu Yu. 2019. "Recovery of Heart Rate Variability After Treadmill Exercise Analyzed by Lagged Poincaré Plot and Spectral Characteristics". figshare. https://hdl.handle.net/2134/26131. 


\section{Recovery of Heart Rate Variability after Treadmill Exercise}

\section{Analyzed by Lagged Poincaré Plot and Spectral}

\section{Characteristics}

Ping Shi ${ }^{1 \S}$, Sijung $\mathrm{Hu}^{2}$, Hongliu $\mathrm{Yu}^{1}$

${ }^{1}$ Institute of Rehabilitation Engineering and Technology, University of Shanghai for

Science and Technology, Shanghai 200093, China

${ }^{2}$ Wolfson School of Mechanical, Electrical and Manufacturing Engineering,

Loughborough University, Leicestershire LE11 3TU UK, United Kingdom

§Corresponding author, Email: garendon@163.com, Phone: +86-21-55271205

- The total number of words of the manuscript: 6213

- The number of words of the abstract: 192

- The number of figures: 3

- The number of tables: 2 
Abstract: The aim of this study was to analysis the recovery of heart rate variability (HRV) after treadmill exercise, and to investigate the autonomic nervous system (ANS) response after exercise. Frequency domain indices, i.e., $\mathrm{LF}\left(\mathrm{ms}^{2}\right), \mathrm{HF}\left(\mathrm{ms}^{2}\right)$, $\mathrm{LF}$ (n.u.), HF(n.u.) and LF/HF, and lagged Poincaré plot width (SD1 $\mathrm{m}$ ) and length (SD2 ${ }_{\mathrm{m}}$ ) were introduced for comparison between the baseline period (Pre-E) before treadmill running and two periods after treadmill running (Post-E1, Post-E2). The correlations between lagged Poincaré plot indices and frequency domain indices were applied to reveal the long range correlation between linear and non-linear indices during the recovery of HRV. The results suggested entirely attenuated autonomic nervous activity to the heart following the treadmill exercise. After the treadmill running, the sympathetic nerves achieved dominance and the parasympathetic activity was suppressed, which lasted for more than 4 mins. The correlations coefficients between lagged Poincaré plot indices and spectral power indices could separate not only Pre-E and two sessions after the treadmill running, but also the two sessions in recovery periods, i.e. Post-E1 and Post-E2. Lagged Poincaré plot as an innovative nonlinear method showed a better performance over linear frequency domain analysis and conventional nonlinear Poincaré plot.

Keywords: Heart rate variability, Autonomic nervous system, Lagged Poincaré plot, Frequency parameter

\section{Introduction}

The evaluation of the recovery of heart rate variability (HRV) is an innovative approach to study the autonomic nervous system (ANS) response after exercise. Exercise intensity, exercise modality, and physiological conditions (e.g. body position) influenced the HRV recovery after exercise [1]. Conventionally, measures of the HRV time- and frequency-domain indices were utilized to examine the underlying physiological control mechanisms of the body during the exercise recovery. The above linear summary measures of HRV displayed a good performance to distinguish the time-varying influence of the ANS and its components on cardiac function during HRV recovery. Kingsley et al. [2], Chen et al. [3] and Teixeira et al. [4] reported the autonomic recovery from acute resistance exercise. In their studies, parasympathetic activity and sympathetic activity at postexercise following acute resistance exercise were investigated using frequency-domain indices of HRV. Sun et al. [5] compared autonomic recovery following an acute bout of treadmill exercise between Chinese and Caucasian using both the time- and frequency-domain HRV indices, which suggested that autonomic recovery was delayed in Chinese following acute treadmill exercise. 
However, non-linear mechanisms are certainly involved in the genesis of HRV, which are largely influenced by complex interactions and variables of haemodynamic, electrophysiological, humoral variables, as well as by autonomic and central nervous regulations [6]. The fact that cardiovascular variability is a result of both linear and nonlinear fluctuations opened new perspectives. Some situations or interventions can change the linear content of the variability, while leaving the nonlinear fluctuations intact. Therefore, methods of nonlinear system dynamics are expected to elicit additional information. Moreover, a combination of linear and non-linear HRV analysis techniques on an automated system is essential for understanding and evaluating cardiac autonomic modulation. Features extracted from both linear and nonlinear algorithms not only show the 'instantaneous' temporal changes in HRV but also describe the underlying physiopathological 'state' of the ANS and the intrinsic nonlinear nature of the autonomically-regulated cardiac rhythm.

Due to the increased use of nonlinear system dynamics in clinical studies, it is very important to understand the physiological mechanisms underlying the generation of these fluctuations. The Poincaré plot is a valuable HRV analysis technique due to its clear interpretation and its ability to display nonlinear aspects of the complex physiological signals [7,6]. The conventional two-dimensional Poincaré plot, i.e. 1-lagged Poincaré plot, is a diagram in which each $R-R$ interval is plotted as a function of the previous one $\left(\left[R R_{i}, R_{i+1}\right]\right)$. The evidence that the next R-R interval is determined from previous beats leads itself to further generalization with different intervals, i.e. m-lagged Poincaré plots $\left(\left[R R_{i}, R R_{i+m}\right], m \geq 1\right)$. As to the lagged Poincaré plot, autocovariance information was incorporated in this novel method, therefore the analysis to various lags can provide new insights into the important role of autonomic control of the heart. The lagged Poincaré plot yields the higher discriminant power in evaluating physiological dynamical characteristics and revealing clinical information that is inaccessible via conventional linear methods [8-11].

Thakre et al. [12] assessed the relation of the heart beats and HRV with different lags in congestive heart failure subjects and normal subjects. They found curvilinear association between lag and Poincaré plot indices in normal subjects but patients. Contreras et al.'s study [13] in assessing correlations between Poincaré plot indices and spectral indices in healthy and diabetic subjects suggested that the correlations between spectral indices and lagged Poincaré widths might be useful to distinguish normal from pathological HRV. However, few studies analysis the autonomic control of the heart in the recovery of HRV following exercise by Lagged Poincaré plots indices. 
The aim of this study was to analysis the recovery of HRV after treadmill exercise, and to assess the influence of time on the recovery of HRV. Moreover, the correlations between lagged Poincaré plot and spectral power indices were studied in three durations before and after the treadmill exercise, which was expected to reveal the long range correlation between linear and non-linear indices during the recovery of HRV, and made further analysis the ANS response after exercise.

\section{Method}

\subsection{Subjects}

Sixteen individuals (8 males and 8 females, age: 23.9 \pm 0.7 years; BMI: $21.5 \pm 2.9 \mathrm{~kg} / \mathrm{m}^{2}$ ) without any history of cardiovascular or neurological disorder volunteered to participate in this study. They all were normotensive, non-smokers, and were not taking any medications at the time of the study. Participants were familiarized with the test procedure and training equipment used in the study for approximately 2 days prior to the data collection. The study protocol was conducted according to the declaration of Helsinki and was approved by the University of Shanghai for Science and Technology Ethical Committee. The contents of the experiment were fully explained in writing to the subjects. All subjects provided written informed consent.

\subsection{Data acquisition system and HRV measurement}

A multi-channel physiological signal recording system (PowerLab 4/26, ADInstruments Pty Ltd., Sydney, Australia) was used to collect data from ECG signals. The lead II ECG signal was recorded with three disposable electrodes placed on the right wrist, the left wrist and the right leg of each subject. The ECG signal was sampled at $1 \mathrm{kHz}$ and recorded directly onto the hard disk of a computer through an analog-digital convertor having a 12 bits resolution. The bandwidth of the ECG amplifiers was set from 0.13 to $100 \mathrm{~Hz}$ with $50 \mathrm{~Hz}$ notch. The values of beat-to-beat cardiac interval were automatically excluded for each sinus beat and were subsequently exported for further analysis using MATLAB (Math-Works Inc., MA, USA). All ECG datasets used for subsequent analysis were free of any form of morphologically abnormal beats.

HRV describes the fluctuation in heartbeat intervals and is a reliable, noninvasive and quantitative marker of ANS activity. HRV characterizes the variations in the time interval between consecutive heartbeats, that is, the variations in duration between the $\mathrm{R}$ peaks on the QRS complexes recognized on ECG tracing. Assessment of HRV is based on analysis of 
fluctuations of heart beat intervals and may provide quantitative information on the modulation of cardiac inputs from parasympathetic and sympathetic nerves. HRV analysis is a well-recognized tool for estimating cardiac autonomic modulations [6].

\subsection{Measurement protocol}

All subjects were instructed to refrain from hot drinks or those containing caffeine and strenuous exercise for $1 \mathrm{~h}$ prior to the study. Subjects rested quietly in a seated position for at least $3 \mathrm{~min}$ before the measurement. Then the resting ECG was recorded $4 \mathrm{~min}$ as the baseline recording (Pre-E). Next, the subjects performed a treadmill running for $8 \mathrm{~min}$ at the speed of 6 $\mathrm{km} / \mathrm{h}$. Immediately following exercise (within $10 \mathrm{~s}$ ), participants were re-seated in a chair and recovered passively with instructions to remain motionless without talking. The ECG was recorded for each subject for 8 min following the treadmill running, which was divided into two periods to do the further processing, including the period of 1-4 min after the treadmill running (Post-E1), and the period of 5-8 min after the treadmill running (Post-E2).

\subsection{Spectral characteristics}

RR interval series extract from ECG tracing is inherently nonuniformly sampled series. Interpolation is necessary to produce a uniformly sampled HRV time series out of an RR interval series. The interpolation frequency was chosen as $4 \mathrm{~Hz}$. After interpolation, the FFT method with Hanning window was used to obtain power spectrum estimates of HRV.

Presently, the investigators have either relied on FFT algorithm or on autoregressive (AR) modeling for spectral estimation of HRV time series. The FFT is easier to implement and is usually employed with a priori selection of the number and frequency range of bands of interest [6]. Conversely, AR algorithms can decompose the overall spectrum into single spectral components, using the residual theorem, thus providing automatically the number, central frequency, and associated power without the need for a priori assumptions [6]. However, by choosing a specific AR model order (ARMO), two different difficulties may arise. Lower orders can interfere in the correct power estimation of the frequency bands and may produce a different effect in each component, mainly in R-R series of high complexity. Higher orders on the other hand are sensible to spurious peaks and produces over fitting [14]. It has been suggested that the order of the model should be fixed instead of being estimated independently for each R-R series $[15,16]$. Recommendations for computational analysis of HRV are not conclusive [6], and 
therefore many controversies still exist about the choice of the optimal model order. Therefore, FFT algorithm was used in the present study for spectral estimation of HRV time series.

The frequency domain HRV variables, gained from a power spectrum of the RR interval series, have been found to reflect autonomic cardiovascular control [6]. Two power components were taken into account in this study: a high frequency (HF: 0.15-0.4 Hz) component and a low frequency (LF: $0.04-0.15 \mathrm{~Hz}$ ) component. HF power was supposedly a marker of parasympathetic activity, and LF power was reflective of both parasympathetic and sympathetic modulation of heart rate [6]. LF/HF ratio was regarded as a mirror of sympathovagal balance or mainly as an index of sympathetic modulations [17,18]. LF and HF were measured as both absolute units (ms2) and normalized units (n.u.). The normalized units were obtained by dividing the power of each component by total power minus the VLF component [6,17]. Normalization could minimize the effect on values of LF and HF components of the changes in total power [6]. Normalized LF and HF, i.e., LF norm and HF norm are expressed as

$$
\begin{aligned}
& \mathrm{LF}(\text { n. u. })=\frac{\mathrm{LF}}{\text { Total Power-VLF }} \times 100 \\
& \mathrm{HF}(\text { n.u. })=\frac{\mathrm{HF}}{\text { Total Power-VLF }} \times 100
\end{aligned}
$$

\subsection{Lagged Poincaré plot}

Poincaré plot outlines the HRV measurements and provide the detailed pulse-to-pulse behavior. Conventional Poincaré plot for HRV analysis is a scatterplot of each RR interval against the previous RR interval, i.e., it has two dimensions and a lag of 1 interval. The geometric appearance of the Poincaré plot can be described by enclosing with an ellipse the points in the plot. The ellipse is fitted onto a new coordinate axis that is rotated $45^{\circ}$ counterclockwise to the normal axis so the new $\mathrm{X}$-axis crosses the ellipse. The standard deviation of the points perpendicular to the new X-axis (SD1) measures the width of the plot and therefore indicates the level of short-term HRV. The standard deviation along the new X-axis (SD2) measures the length of the plot and is thought to indicate the level of long-term HRV. The present quantitative analysis was planned to measure SDl, SD2 and SD1/SD2 ratio, respectively. Generally, SD1 is the parameter of parasympathetic control of the sinus node [19, 20]. SD2 is influenced by both parasympathetic and sympathetic tone [19-22]. SD1/SD2 ratio could be used as an indicator of sympathetic activity [22].

Instead of the plotting $R R_{\mathrm{n}}$ against $R R_{\mathrm{n}+1}$ in conventional two-dimensional Poincaré plots , i.e., 1-lagged Poincaré plots, generalization of Poincaré plots by plotting $m$-lagged plots was investigated in this study, where $m$ is allowed to vary from 1 to some small positive value. M- 
lagged Poincaré plots, plotting $R R_{\mathrm{n}}$ against $R R_{\mathrm{n}+\mathrm{m}}$, assume the previous interval can affect multiple successive intervals. The length and the width of the m-lagged Poincaré plot are altered as the lag is increased. SD1 and SD2 generalized for lag $m$ are given by [23].

$$
\begin{aligned}
& S D 1(m)^{2}=\phi_{P P}(0)-\phi_{P P}(m) \\
& S D 2(m)^{2}=\phi_{P P}(0)+\phi_{P P}(m)
\end{aligned}
$$

Where $\phi_{R R}(m)$ is the mean-removed autocorrelation function, i.e., autocovariance function, and is expressed as

$$
\phi_{P P}(m)=\mathrm{E}\left[\left(P P_{\mathrm{n}}-\overline{P P}\right)\left(P P_{n+m}-\overline{P P}\right)\right]
$$

Considering the facts that $m$-lagged Poincaré plots can be described as the autocovariance function, and the autocovariance function monotonically decreases with the increasing lag for values of lag less than 10, lags between 1 and 10 for each subject were applied in this study. $\mathrm{SD} 1_{\mathrm{m}}$ and $\mathrm{SD} 2_{\mathrm{m}}$ are introduced to express the width and the length in $m$-lagged Poincaré plots, respectively.

In this study, correlation coefficients $(r)$ between the frequency-domain HRV and the lagged Poincaré plot indices were accounted for within three measurement periods e.g. Pre-E, PostE1and Post-E2.

\subsection{Statistics}

Normal distribution of each index was assessed applying the Kolmogorov-Smirnov test revealing a normal distribution for all HRV indices. Variables in this study were given as mean \pm SD. The significance of difference between sessions was compared using one-way ANOVA with repeated-measures followed by post hoc analysis. A $\mathrm{p}<0.05$ was considered statistically significant. The statistical analyses were run in MATLAB software (MathWorks Inc., MA,USA).

\section{Results}

\subsection{Frequency domain indices in the recovery of $\mathrm{HRV}$}

In Table 1, $\mathrm{LF}\left(\mathrm{ms}^{2}\right)$ and $\mathrm{HF}\left(\mathrm{ms}^{2}\right)$ were observed significantly decreased during Post-E1 and Post-E2 compared to the baseline recording Pre-E. HF(n.u.) significantly decreased, and LF(n.u.) and LF/HF significantly increased during recovering time. However, there were no significant differences between Post-E1 and Post-E2 for five frequency domain indices. The trends of the 
change of frequency domain indices for Post-E1 and Post-E2 were in the direction of Pre-E, but did not return to the baseline level.

\subsection{Lagged Poincaré plots indices in the recovery of HRV}

In Table1, the differences between Post-E1 and Post-E2 cannot be distinguished. Therefore, the lagged Poincaré plots, which studied the long term variability of HRV, were applied to examine the difference between Post-E1 and Post-E2.

In Table 2, SD1 $\mathrm{m}$ in Post-E1 and Post-E2 were significantly decreased for all lags compared with Pre-E. However, there were no significant differences between Post-E1 and Post-E2 for $\mathrm{SD} 1_{\mathrm{m}} . \mathrm{SD} 2_{\mathrm{m}}$ in Post-E1 and Post-E2 were significantly decreased for all lags compared with PreE. $\mathrm{SD} 2 \mathrm{~m}$ with lag 5 and 6 in Post-E2 were significantly decreased compared with Post-E1. $\mathrm{SD} 1_{\mathrm{m}} / \mathrm{SD} 2_{\mathrm{m}}$ in Post-E1 were significantly decreased compared with Pre-E. SD $1_{\mathrm{m}} / \mathrm{SD} 2_{\mathrm{m}}$ with lag 5 , 6 and 7 in Post-E2 were significantly decreased compared with Post-E1.

\subsection{Correlation between lagged Poincaré plot indices and frequency domain indices}

This study examined the correlation between lagged Poincaré plot indices and frequency domain indices during Pre-E, Post-E1 and Post-E2 to look into the ANS response after exercise.

In Fig. 1, the correlation coefficients between $\mathrm{SD} 1_{\mathrm{m}}$ and $\mathrm{LF}$ component in Pre-E were lower than those in Post-E1 and Post-E2. The correlation coefficients between SD1 $\mathrm{m}$ and LF(n.u.) component in Pre-E were lower than those in Post-E1 and Post-E2. The correlation coefficients between SD1 ${ }_{m}$ and HF(n.u.) component in Pre-E were higher than those in Post-E1 and Post-E2. The correlation coefficients between $\mathrm{SD} 1_{\mathrm{m}}$ and $\mathrm{LF} / \mathrm{HF}$ in Pre-E were lower than those in Post-E1 and Post-E2.

In Fig. 2, Pre-E, Post-E1, and Post-E2 were separated by the correlation coefficients between $\mathrm{SD} 2 \mathrm{~m}$ and frequency domain indices. In Fig. 3, the results for the correlation coefficients between frequency indices and $\mathrm{SD} 1_{\mathrm{m}} / \mathrm{SD} 2_{\mathrm{m}}$ indicated that lag 4 deserved attentions. For example, the correlation coefficients between $\mathrm{LF}\left(\mathrm{ms}^{2}\right)$ and $\mathrm{SD} 1_{\mathrm{m}} / \mathrm{SD} 2_{\mathrm{m}}$ in Pre-E gradually rose for the lag $<4$, but declined for the lag $>4$ (Fig. 3a). The results indicated that lag 4 is the key point for the change of coefficients.

Generally, Pre-E, Post-E1, and Post-E2 could be visually distinguished by the correlation coefficients between lagged Poincaré plot indices and frequency domain indices.

\section{Discussion}




\subsection{Frequency domain indices in the recovery of $H R V$}

Evaluation of heart rate recovery after exercise has become a valuable, noninvasive procedure to assess cardiovascular-para-sympathetic influence [24, 25]. Time-domain was considered a less measure of cardiac modulation of ANS [6]. In this study, frequency indices LF, HF and LF/HF were used to evaluate the cardio-physiological behavior in Pre-E, Post-E1 and Post-E2. Spectral analysis of HRV is a useful, noninvasive technique to investigate the short-term (2-5 min) autonomic modulation of heart rate under physiologically stable conditions [6]. For the frequency-domain indices of HRV, the HF component has been linked to the activity of parasympathetic nervous system, while the LF component is reflective of both sympathetic modulation and parasympathetic tone [26-28]. LF/HF ratio was considered as an index of parasympathetic and sympathetic interactions, i.e., the index of sympathovagal balance [26,27]. In this study, significantly decreased $\mathrm{HF}\left(\mathrm{ms}^{2}\right)$ and $\mathrm{HF}$ (n.u.) indicated that the parasympathetic activity was suppressed after treadmill exercise. The decreased $\mathrm{LF}\left(\mathrm{ms}^{2}\right)$ after the treadmill exercise suggested the suppressed sympathetic activity and parasympathetic tone due to exercise. However, accumulating evidence demonstrated that the interpretation about power spectral analysis over simplifies the complex non-linear interactions between the sympathetic and the parasympathetic divisions of the ANS [29-32].

Despite serious and largely under-appreciated limitations, the LF/HF ratio has gained wide acceptance as a tool to assess cardiovascular autonomic regulation where increases in LF/HF are assumed to reflect a shift to "sympathetic dominance" and decreases in this index correspond to a “parasympathetic dominance.” In the present study, significant increased LF(n.u.) and LF/HF in Post-E1 and Post-E2 demonstrated that the sympathetic nerves achieved dominance over the parasympathetic nerves following the treadmill exercise, although the dominance diminished over time during the recovery of HRV. The phenomenon in this study indicated that LF/HF was tended to calculate as an autonomic-imbalance index, which was coincident with the claim from Billman's paper. Billman [33] also indicated that the physiological basis for LF/HF was difficult to discern due to the disagreement in interpretation of the LF and HF component of HRV.

The changes in five frequency domain measures between Pre-E and two sessions after treadmill running suggested entirely attenuated autonomic nervous activity to the heart, and may reflect diminished afferent autonomic activity following the treadmill exercise. However, frequency domain measures cannot distinguish the difference between Post-E1 and Post-E2. It is possible that the intrinsic nonlinear nature of the autonomically regulated cardiac rhythm implies the linear data processing techniques do not predict the subtle change of the ANS. 


\subsection{Lagged Poincaré plots indices in the recovery of HRV}

Poincaré plot is one of the most powerful nonlinear tools for qualitative exploration the dynamics of physiological signal. Previous studies have shown the evaluation of the conventional Poincaré plot for HRV analysis in investigating the function of ANS. It has been shown that SD1 correlates better with HF component than with LF component [7,23]. Brennan et al.[23] suggested that conventional Poincaré plot ignored some of its most potent abilities to display nonlinear aspects of the interval sequence while converting the two-dimensional plot into various one-dimensional views which existing HRV indexes already specify. They expressed that the set of lagged Poincaré plots could be a complete and better description of the autocovariance function and the power spectrum of the HRV intervals [23]. In the present study, we have assessed how these correlations were affected when SD1 and SD2 was obtained for longer lags (m>1) during HRV recovery after treadmill exercise. Lagged Poincaré plots in this study provided information about the cardiac response for the recovery of HRV following treadmill exercise in different sessions that was less evident in the linear-based methods.

Table 2 showed that all lagged Poincaré plot measures significantly changed in both Post-E1 and Post-E2 compared to these in Pre-E. The changes between Post-E1 and Post-E2 were distinguished while the lag was 5, 6 and 7. It was demonstrated that lagged Poincaré plot have sufficient sensitivity in detecting the obscure changes in ANS, that is not visible from frequencydomain indices, and conventional Poincaré plot as well. It has been reported that a heartbeat influences not only the beat immediately, but also up to 4-10 beats downstream [34], which could be due to the variation in heart rate as a consequence of respiration, i.e., respiratory sinus arrhythmia.

Goshvarpour et al. indicated that indices in Poincaré plot with lag 6 were found comparative while studying the nonlinear behavior of physiologic signals [35]. Several studies showed that Poincaré plot with increased lags had better performance in predicting pathophysiological risk or accessing physiological condition [8-11]. In the present study, we speculated that the respiratory rhythm greatly affects the recovery of HRV at different durations after exercise. The influence of the respiratory rhythm was presented in all lagged Poincaré plot, particularly while the lag is 5, 6 and 7.

In practically, behavioural factors influence the HRV and HRV recovery, including physical activity, smoking habits, alcohol consumption and dietary patterns. For example, regular physical activity has been shown to lower resting HR [36]; to improve HR recovery after acute exercise[33, 
34] and to increase global HRV[38-40] due to physical activity causes a resting bradycardia. However, literatures support the interpretation that there may be no true causal association of behavioural factors with HRV [41, 42]. Therefore, the questionnaire about the behavioural factors for the participants was not conducted in this study. Many researches indicated that exercise duration, intensity and mode may affect the post-exercise recovery of physiological parameters in acute exercise [43-45]. However, the above studies always provided the temporal or spectral mathematical approaches, which performed well in stationary segments of the signal, yet neglected the nonlinear characteristic aspects of RR interval. Lagged Poincaré plot as a novel technology allowed researches to analyze the HRV in non-stationary condition, such as assessment of the cardiac autonomic activity at the immediate post-exercise period in this study.

As suggested by previous studies, the width of Poincaré plot (SD1) could be considered as a nonlinear indicator of parasympathetic activity, while the length of Poincaré plot (SD2) is influenced by both sympathetic and parasympathetic tone [21,22]. SD1/SD2 ratio could be used as a marker of sympathetic activity since it increased during the exercise after a complete parasympathetic blockade. $\mathrm{SD} 2_{\mathrm{m}}$ and $\mathrm{SD} 1_{\mathrm{m}} / \mathrm{SD} 2_{\mathrm{m}}$ ratio continued to decrease in Post-E2 and the significant differences were detected at lag 5, 6 and 7, which suggested that the entirely attenuated autonomic nervous activity to the heart and the inhibition of sympathetic activity lasted for more than 4 mins.

\subsection{Correlation between lagged Poincaré plot indices and frequency domain indices}

A combination of linear and nonlinear HRV analysis techniques are applied widely to monitor the changes of automated system and would offer valuable insight into ANS functioning and response $[46,47]$. The correlation between lagged Poincaré plot indices and spectral power indices may uncover abnormalities that are not easily detectable with frequency domain measures. In the present study, the results indicated that the combination of lagged Poincaré plot and frequency domain measures could separate not only Pre-E and two sessions following treadmill running, but also the two recovery periods, i.e. Post-E1 and Post-E2.

An established physiological oscillator model have expressed that the HF component contributes greatly to the width of Poincaré plot, and that the LF component contribution was relatively minor [7]. The LF/ HF significantly correlated with SD2 [48]. However, the above statement was based on the conventional Poincaré plot (lag=1). As to the lagged Poincaré plot (lag>1), with the augment of the lags, there was no general agreement on how LF or HF components contribute to the width and the length of lagged Poincaré plot. The results in this study indicated that, in the correlation between lagged Poincaré plot indices and frequency 
domain indices, medium-term (lag=4) inter-beat correlation could be a valuable index, which may be associated with the respiratory sinus arrhythmia. Since the two branches of the ANS i.e., sympathetic and parasympathetic nervous systems, are reciprocally innervated, their responses are co-ordinated to provide the appropriate internal environment to meet shifts in both internal and external demands. Therefore, in this study, the recovery time and the respiratory sinus arrhythmia were both considered as the factors that influenced the recovery of HRV. Wessel et al [49] gave an example of cardiorespiratory modeling which demonstrates that the complex behaviour of the heart rate is mainly caused by the respiration which influences the coupling of heart rate and blood pressure. It is known that respiration is not perfectly periodic and even a resting person experiences irregular breathing due to several factors. Therefore, the influence of respiration on HRV would be expected to be in both LF and HF [50]. Lagged Poincaré plot expected to provide the opportunity to explore the nature of nonlinear physiological response due to its physiological correlation with HF and LF.

\section{Conclusion}

Generally, lagged Poincaré plot as an innovative nonlinear method showed a clear advantage over linear frequency domain indices and conventional nonlinear Poincaré plot. The present study indicated that nonlinearity in the cardiovascular system cannot be reflected by standard HRV analysis. The experiment results show the effectiveness of the lagged Poincaré plot method and its correlation with frequency domain indexes of HRV for assessing the recovery of HRV after treadmill exercise. The autonomic nervous activity attenuated following the treadmill exercise, and the sympathetic nerves achieved dominance after the treadmill running.

The goal is not that nonlinear HRV techniques would replace the conventional linear analysis, but they have to be considered as an addition, yielding the nonlinearity and possible chaos of the cardiovascular system.

\section{Competing interests statement}

None declared.

\section{Acknowledgment}

This work was supported by Innovation Program of Shanghai Municipal Education Commission (Grant no. 14YZ091). 


\section{Ethical approval}

All procedures performed in studies involving human participants were in accordance with the ethical standards of the institutional and/or national research committee and with the 1964 Helsinki Declaration and its later amendments or comparable ethical standards. The study was approved by the ethics committee of University of Shanghai for Science and Technology, Shanghai, China (Ref. No. 2013-9023-14YZ091).

\section{References}

1. Casonatto J, Tinucci T, Dourado AC, Polito M (2011) Cardiovascular and autonomic responses after exercise sessions with different intensities and durations. Clinics (Sao Paulo) 66(3):453-458

2. Kingsley JD, Hochgesang S, Brewer A, Buxton E, Martinson M, Heidner G (2014) Autonomic modulation in resistance-trained individuals after acute resistance exercise. Int J Sports Med 35(10):851-856

3. Chen J-L, Yeh D-P, Lee J-P, Chen C-Y, Huang C-Y, Lee S-D, Chen C-C, Kuo TBJ, Kao C-L, Kuo C-H (2011) Parasympathetic nervous activity mirrors recovery status in weightlifting performance after training. J Strength Cond Res 25(6):1546-1552

4. Teixeira L, Ritti-Dias RM, Tinucci T, Mion Junior D, Forjaz CLdM (2011) Postconcurrent exercise hemodynamics and cardiac autonomic modulation. Eur J Appl Physiol 111(9):2069-2078

5. Sun P, Yan H, Ranadive SM, Lane AD, Kappus RM, Bunsawat K, Baynard T, Hu M, Li S, Fernhall B (2016) Autonomic recovery is delayed in chinese compared with caucasian following treadmill exercise. PLoS One 11(1):e0147104

6. Task force of the european society of cardiology and the north american society of pacing and electrophysiology (1996) Heart rate variability: Standards of measurement, physiological interpretation and clinical use. Circulation 93(5):1043-1065

7. Brennan M, Palaniswami M, Kamen P (2002) Poincaré plot interpretation using a physiological model of hrv based on a network of oscillators. Am J Physiol Heart Circ Physiol 283(5):H1873-1886

8. Karmakar C, Jelinek HF, Khandoker A, Tulppo M, Makikallio T, Kiviniemi A, Huikuri H, Palaniswami M (2015) Multi-lag HRV analysis discriminates disease progression of post-infarct people with no diabetes versus diabetes. Conf Proc IEEE Eng Med Biol Soc 2015:2367-2370

9. Nardelli M, Valenza G, Greco A, Lanata A, Scilingo EP (2015) Arousal recognition system based on heartbeat dynamics during auditory elicitation. Conf Proc IEEE Eng Med Biol Soc 2015:6110-6113

10. Roy B, Ghatak S (2013) Nonlinear methods to assess changes in heart rate variability in type 2 diabetic patients. Arq Bras Cardiol 101(4):317-327 
11. Voss A, Fischer C, Schroeder R, Figulla HR, Goernig M (2012) Lagged segmented Poincaré plot analysis for risk stratification in patients with dilated cardiomyopathy. Med Biol Eng Comput 50(7):727-736

12. Thakre TP, Smith ML (2006) Loss of lag-response curvilinearity of indices of heart rate variability in congestive heart failure. BMC Cardiovasc Disord 6:27

13. Contreras P, Canetti R, Migliaro ER (2007) Correlations between frequency-domain HRV indices and lagged poincaré plot width in healthy and diabetic subjects. Physiol Meas 28(1):85-94

14. Takalo R, Hytti H, Ihalainen H (2005)Tutorial on univariate autoregressive spectral analysis. J Clin Monit Comput 19(6):401-410.

15. Boardman A, Schlindwein FS, Rocha AP, Leite A (2002)A study on the optimum order of autoregressive models for heart rate variability. Physiol Meas 23(2):325-336.

16. Tarvainen MP, Georgiadis SD, Ranta-Aho PO, Karjalainen PA (2006)Time-varying analysis of heart rate variability signals with a Kalman smoother algorithm. Physiol Meas 27(3):225-239.

17. Pagani M, Lombardi F, Guzzetti S, Rimoldi O, Furlan R, Pizzinelli P, Sandrone G, Malfatto G, Dell'Orto S, Piccaluga E (1986) Power spectral analysis of heart rate and arterial pressure variabilities as a marker of sympatho-vagal interaction in man and conscious dog. Circ Res 59(2):178-193

18. Eckberg DL (1997) Sympathovagal balance: A critical appraisal. Circulation 96(9):32243232

19. Kamen PW, Krum H, Tonkin AM (1996) Poincare plot of heart rate variability allows quantitative display of parasympathetic nervous activity in humans. Clin Sci (Lond) 91(2):201-208

20. Kamen PW, Tonkin AM (1995) Application of the Poincare plot to heart rate variability: a new measure of functional status in heart failure. Aust N Z J Med 25(1):18-26

21. De Vito G, Galloway SDR, Nimmo MA, Maas P, McMurray JJV (2002) Effects of central sympathetic inhibition on heart rate variability during steady-state exercise in healthy humans. Clin Physiol Funct Imaging 22(1):32-38

22. Tulppo MP, Makikallio TH, Takala TE, Seppanen T, Huikuri HV (1996) Quantitative beat-to-beat analysis of heart rate dynamics during exercise. Am J Physiol 271(1 Pt 2):H244-252

23. Brennan M, Palaniswami M, Kamen P (2001) Do existing measures of Poincaré plot geometry reflect nonlinear features of heart rate variability? IEEE Trans Biomed Eng 48(11):1342-1347

24. Imai K, Sato H, Hori M, Kusuoka H, Ozaki H, Yokoyama H, Takeda H, Inoue M, Kamada T (1994) Vagally mediated heart rate recovery after exercise is accelerated in athletes but blunted in patients with chronic heart failure. J Am Coll Cardiol 24(6):15291535

25. Mendonca GV, Pereira FD, Fernhall B (2013) Heart rate recovery and variability following combined aerobic and resistance exercise training in adults with and without down syndrome. Res Dev Disabil 34(1):353-361

26. Burr RL (2007) Interpretation of normalized spectral heart rate variability indices in sleep research: A critical review. Sleep. 30(7):913-919

27. Malliani A, Pagani M, Lombardi F, Cerutti S (1991) Cardiovascular neural regulation explored in the frequency domain. Circulation 84(2):482-492

28. Miyata TT, Oishi S, Taniguchi Y, Kawai H, Yasaka Y, Yokoyama H (2016) Power spectral analysis of heart rate variability during the postural change from sitting to upright position reveal sympathetic hyperactivity. Eur J Heart Fail 18(S1):407-407 
29. Lombardi F, Stein PK (2011) Origin of heart rate variability and turbulence: an appraisal of autonomic modulation of cardiovascular function. Front Physiol 2:95

30. Francesco B, Maria Grazia B, Emanuele G, Valentina F, Sara C, Chiara F, Riccardo M, Francesco F (2012) Linear and nonlinear heart rate variability indexes in clinical practice. Comput Math Methods Med 2012:219080

31. Billman GE (2011) Heart rate variability - a historical perspective. Front Physiol 2:86.

32. Dantas EM, Andreao RV, da Silva VJ, Ribeiro AL, Kemp AH, Brunoni AR, Lotufo PA, Rodrigues SL, Bensenor IM, Mill JG (2015) Comparison between symbolic and spectral analyses of short-term heart rate variability in a subsample of the ELSA-Brasil study. Physiol Meas 36(10):2119-2134

33. Billman GE (2013) The LF/HF ratio does not accurately measure cardiac sympatho-vagal balance. Frontiers in physiology 4:26

34. Lerma C, Infante O, Perez-Grovas H, Jose MV (2003) Poincaré plot indexes of heart rate variability capture dynamic adaptations after haemodialysis in chronic renal failure patients. Clin Physiol Funct Imaging 23(2):72-80

35. Goshvarpour A, Goshvarpour A (2015) Poincaré indices for analyzing meditative heart rate signals. Biomed J 38(3):229-234

36. Carter JB, Banister EW, Blaber AP (2003) Effect of endurance exercise on autonomic control of heart rate. Sports Med 33(1):33-46

37. Figueroa A, Baynard T, Fernhall B, Carhart R, Kanaley JA (2007) Endurance training improves post-exercise cardiac autonomic modulation in obese women with and without type 2 diabetes. Eur J Appl Physiol 100(4):437-444

38. Levy WC, Cerqueira MD, Harp GD, Johannessen KA, Abrass IB, Schwartz RS, Stratton JR (1998) Effect of endurance exercise training on heart rate variability at rest in healthy young and older men. Am J Cardiol 82(10):1236-1241

39. Jurca R, Church TS, Morss GM, Jordan AN, Earnest CP (2004) Eight weeks of moderate-intensity exercise training increases heart rate variability in sedentary postmenopausal women. Am Heart J 147(5):e21

40. Madden KM, Levy WC, Stratton JK: Exercise training and heart rate variability in older adult female subjects. Clin Invest Med 2006, 29(1):20-28

41. Panda K, Krishna P (2014) Physical exercise and cardiac autonomic activity in healthy adult men. Indian J Physiol Pharmacol 58(4):365-370

42. Kluttig A, Schumann B, Swenne CA, Kors JA, Kuss O, Schmidt H, Werdan K, Haerting J, Greiser KH (2010) Association of health behaviour with heart rate variability: a population-based study. BMC cardiovascular disorders 10:58

43. Figueiredo T, Willardson JM, Miranda H, Bentes CM, Reis VM, Simao R (2015) Influence of Load Intensity on Postexercise Hypotension and Heart Rate Variability after a Strength Training Session. J Strength Cond Res 29(10):2941-2948

44. Saboul D, Balducci P, Millet G, Pialoux V, Hautier C (2016) A pilot study on quantification of training load: The use of HRV in training practice. Eur J Sport Sci 16(2):172-181.

45. Stoggl T, Schwarzl C, Muller EE, Nagasaki M, Stoggl J, Schonfelder M, Niebauer J (2017) Alpine Skiing as Winter-Time High-Intensity Training. Med Sci Sports Exerc. doi: 10.1249/MSS.0000000000001289.

46. Weippert M, Behrens K, Rieger A, Kumar M, Behrens M (2015) Effects of breathing patterns and light exercise on linear and nonlinear heart rate variability. Appl Physiol Nutr Metab 40(8):762-768

47. Zhang D, She J, Yang J, Yu M (2015) Linear and nonlinear dynamics of heart rate variability in the process of exposure to 3600m in 10min. Australas Phys Eng Sci Med 38(2):263-270 
48. Guzik P, Piskorski J, Krauze T, Schneider R, Wesseling KH, Wykretowicz A, Wysocki $\mathrm{H}$ (2007) Correlations between the Poincaré plot and conventional heart rate variability parameters assessed during paced breathing. J Physiol Sci 57(1):63-71

49. Wessel N, Riedl M, Kurths J (2009) Is the normal heart rate "chaotic" due to respiration? Chaos 19(2):028508

50. Wessel N, Sidorenko L, Kraemer JF, Schoebel C, Baumann G (2016) Assessing cardiac autonomic function via heart rate variability analysis requires monitoring respiration. Europace 18(8):1280 
Table 1: Comparisons from frequency domain between Pre-E, Post-E1 and Post-E2. LF: low-frequency power; HF: high-frequency power; LF (n.u.): normalized HRV indices [LF/(LF + HF)]; HF (n.u.): normalized HRV indices [HF/(LF + HF)]; LF/HF: the power ratio of low-frequency to high-frequency. Values are given as mean \pm SD.

\begin{tabular}{c|cccccc}
\hline & Pre-E & Post-E1 & Post-E2 & $p 1$ & $p 2$ & $p 3$ \\
\hline LF(ms $)$ & $1129.4 \pm 547.9$ & $433.4 \pm 168.9$ & $443.2 \pm 186.2$ & $<0.001$ & $<0.05$ & 0.47 \\
LF(n.u.) & $58.1 \pm 21.0$ & $70.4 \pm 10.6$ & $68.1 \pm 18.0$ & $<0.001$ & $<0.05$ & 0.22 \\
HF(ms $\left.{ }^{2}\right)$ & $644.4 \pm 198.2$ & $65.4 \pm 21.7$ & $98.1 \pm 47.5$ & $<0.001$ & $<0.001$ & 0.13 \\
HF(n.u.) & $30.1 \pm 11.4$ & $14.3 \pm 5.3$ & $16.5 \pm 7.6$ & $<0.001$ & $<0.05$ & 0.16 \\
LF/HF & $2.62 \pm 1.1$ & $6.45 \pm 2.8$ & $5.19 \pm 2.3$ & $<0.05$ & $<0.05$ & 0.17 \\
\hline
\end{tabular}

p1: Pre-E v.s. Post-E1; p2: Pre-E v.s. Post-E2; p3: Post-E1 v.s. Post-E2 
Table 2. Summary of lagged Poincaré plot indices in Pre-E, Post-E1 and Post-E2.

\begin{tabular}{|c|c|c|c|c|c|c|c|c|c|}
\hline & \multicolumn{3}{|c|}{ SD1m(ms) } & \multicolumn{3}{|c|}{$\mathrm{SD} 2 \mathrm{~m}(\mathrm{~ms})$} & \multicolumn{3}{|c|}{$\mathrm{SD} 1 \mathrm{~m} / \mathrm{SD} 2 \mathrm{~m}$} \\
\hline Lag & Pre-E & Post-E1 ${ }^{\mathrm{a}}$ & Post-E2 ${ }^{b}$ & Pre-E & Post-E1 ${ }^{\mathrm{a}}$ & Post-E2 ${ }^{b}$ & Pre-E & Post-E1 ${ }^{\mathrm{a}}$ & Post-E2 \\
\hline 1 & $29.1 \pm 14.9$ & $11.2 \pm 5.5$ & $14.4 \pm 7.6$ & $75.4 \pm 23.3$ & $50.4 \pm 15.4$ & $43.8 \pm 17.7$ & $0.37 \pm 0.11$ & $0.23 \pm 0.10$ & $0.34 \pm 0.11$ \\
\hline 2 & $38.3 \pm 17.4$ & $14.3 \pm 6.9$ & $17.9 \pm 8.9$ & $71.1 \pm 21.4$ & $49.5 \pm 14.9$ & $43.6 \pm 16.9$ & $0.52 \pm 0.12$ & $0.29 \pm 0.11$ & $0.41 \pm 0.09$ \\
\hline 3 & $43.5 \pm 18.3$ & $17.0 \pm 9.1$ & $20.7 \pm 10.4$ & $68.2 \pm 20.2$ & $48.5 \pm 14.3$ & $41.3 \pm 15.9$ & $0.62 \pm 0.11$ & $0.36 \pm 0.14$ & $0.48 \pm 0.10$ \\
\hline 4 & $45.8 \pm 18.4$ & $19.2 \pm 11.1$ & $22.9 \pm 11.9$ & $66.7 \pm 20.1$ & $47.5 \pm 13.6$ & $40.1 \pm 14.9$ & $0.67 \pm 0.12$ & $0.41 \pm 0.17$ & $0.55 \pm 0.12$ \\
\hline 5 & $48.4 \pm 17.7$ & $21.4 \pm 12.6$ & $25.3 \pm 12.8$ & $64.8 \pm 20.7$ & $46.3 \pm 13.0$ & $38.6 \pm 14.2 *$ & $0.74 \pm 0.14$ & $0.46 \pm 0.21$ & $0.63 \pm 0.14^{*}$ \\
\hline 6 & $50.4 \pm 16.9$ & $23.2 \pm 13.5$ & $27.3 \pm 13.3$ & $63.2 \pm 21.8$ & $45.3 \pm 12.7$ & $37.2 \pm 13.8^{*}$ & $0.81 \pm 0.18$ & $0.52 \pm 0.23$ & $0.71 \pm 0.16^{*}$ \\
\hline 7 & $51.1 \pm 16.4$ & $24.4 \pm 13.8$ & $28.6 \pm 13.7$ & $62.4 \pm 22.7$ & $44.6 \pm 12.6$ & $36.2 \pm 13.5$ & $0.85 \pm 0.24$ & $0.56 \pm 0.24$ & $0.77 \pm 0.17^{*}$ \\
\hline 8 & $50.7 \pm 15.6$ & $25.1 \pm 13.8$ & $29.1 \pm 13.9$ & $62.5 \pm 23.9$ & $44.2 \pm 12.6$ & $35.8 \pm 13.3$ & $0.87 \pm 0.34$ & $0.58 \pm 0.25$ & $0.79 \pm 0.17$ \\
\hline 9 & $51.2 \pm 16.2$ & $25.2 \pm 13.5$ & $29.3 \pm 14.1$ & $61.9 \pm 24.1$ & $44.2 \pm 12.7$ & $35.6 \pm 13.1$ & $0.91 \pm 0.45$ & $0.58 \pm 0.24$ & $0.80 \pm 0.18$ \\
\hline 10 & $51.6 \pm 16.4$ & $25.2 \pm 12.9$ & $29.4 \pm 14.2$ & $61.4 \pm 24.5$ & $44.3 \pm 13.1$ & $35.5 \pm 13.1$ & $0.96 \pm 0.62$ & $0.58 \pm 0.24$ & $0.81 \pm 0.18$ \\
\hline
\end{tabular}

SD1m: the width of m-lagged Poincaré plot, SD2m: the length of m-lagged Poincaré plot.

${ }^{\text {a }} p<0.05$ for $\mathrm{SD} 1_{\mathrm{m}}(\mathrm{m}=1,2,3 \ldots, 10)$ in Post-E1 compared with Pre-E.

${ }^{\mathrm{b}} p<0.05$ for $\mathrm{SD} 2_{\mathrm{m}}(\mathrm{m}=1,2,3 \ldots, 10)$ in Post-E2 compared with Pre-E.

$* p<0.05$ for SD2m (m=5, 6, and 7) in Post-E2 compared with Post-E1. 


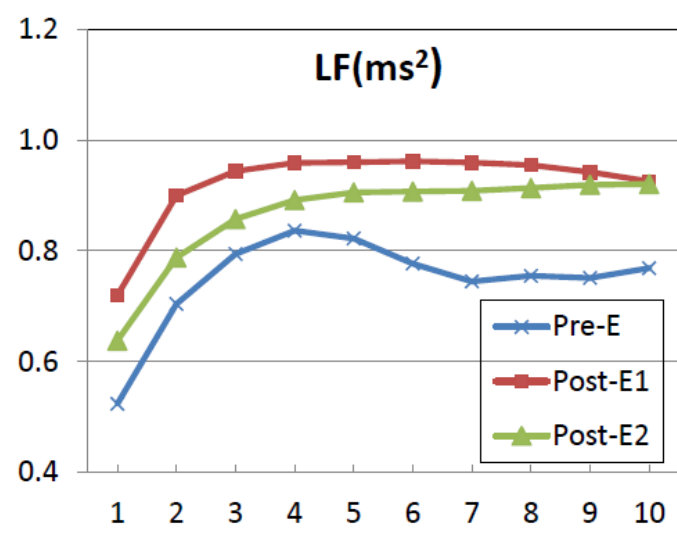

(a)

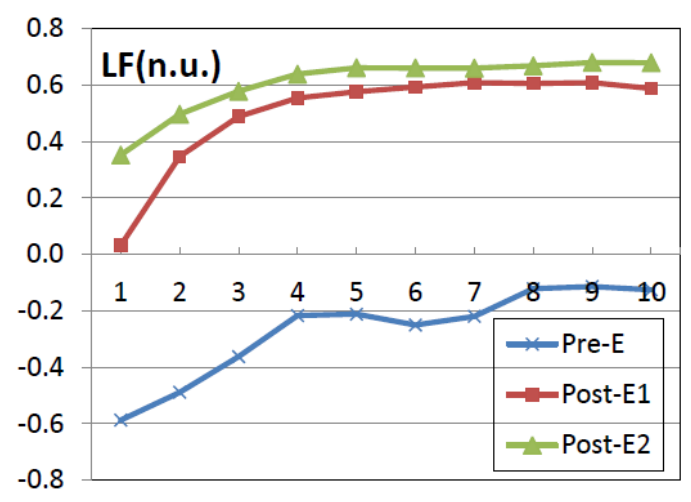

(c)

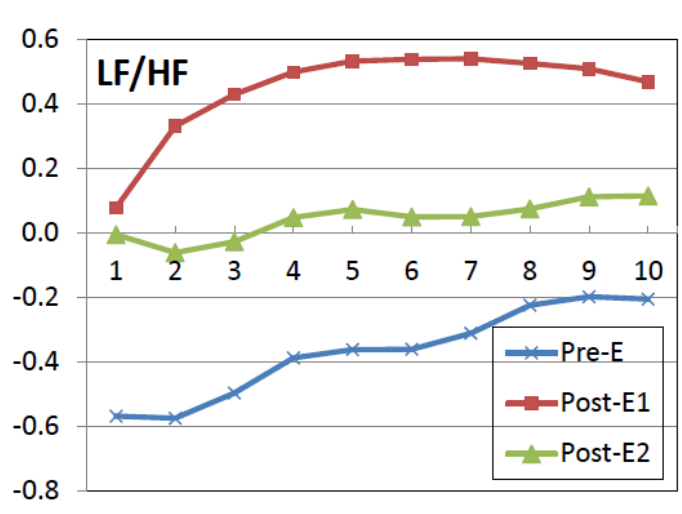

(e)

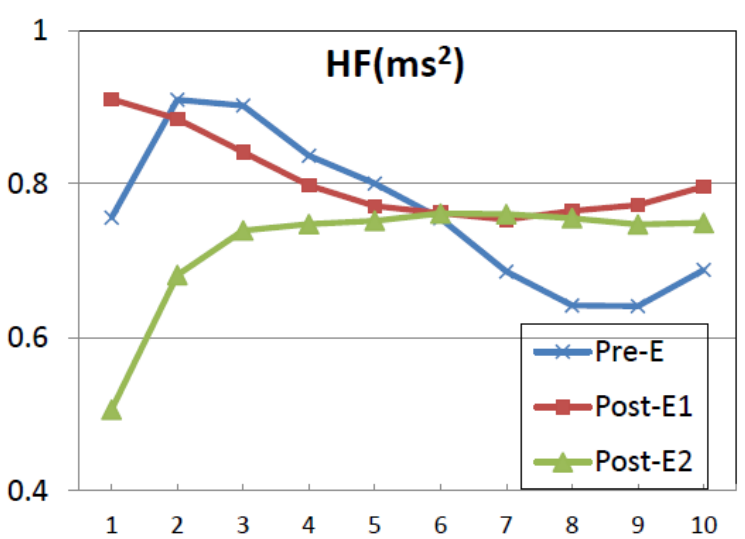

(b)

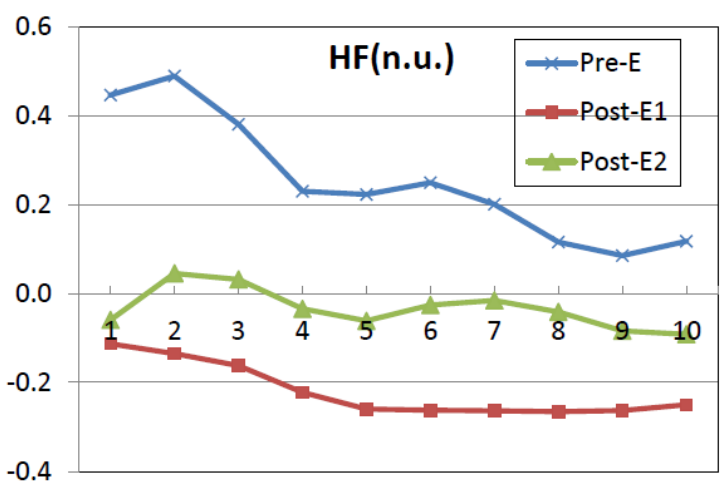

(d)

Fig. 1. The correlations between $S D 1_{m}$ and frequency domain indices: (a) $\mathrm{LF}\left(\mathrm{ms}^{2}\right)$, (b) $\mathrm{HF}\left(\mathrm{ms}^{2}\right)$, (c) LF(n.u.), (d) HF(n.u.), and (e) LF/HF. 


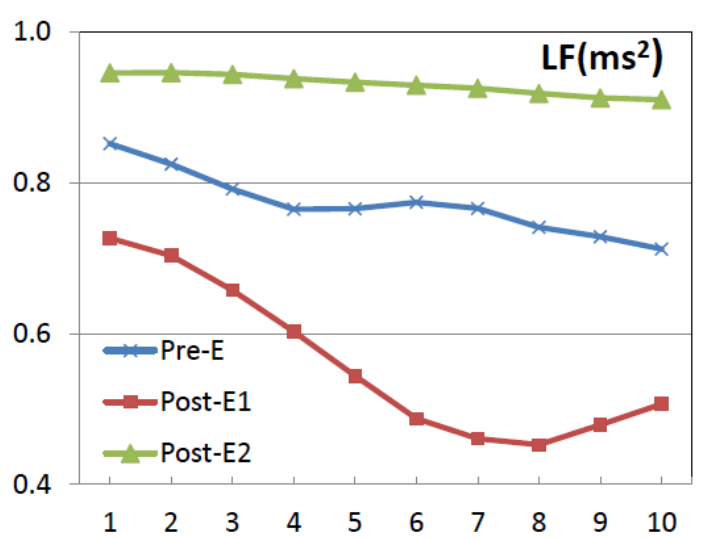

(a)

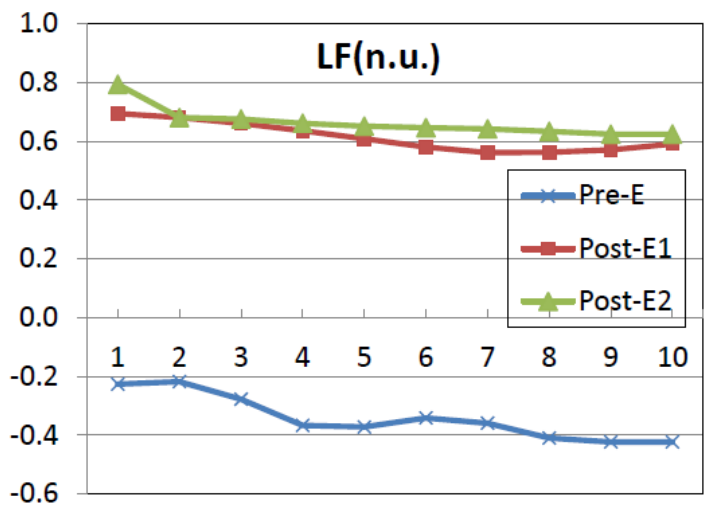

(c)

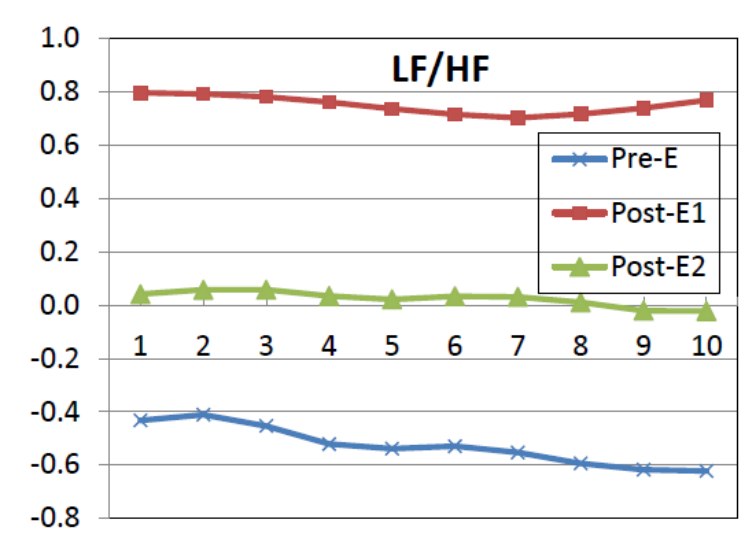

(e)

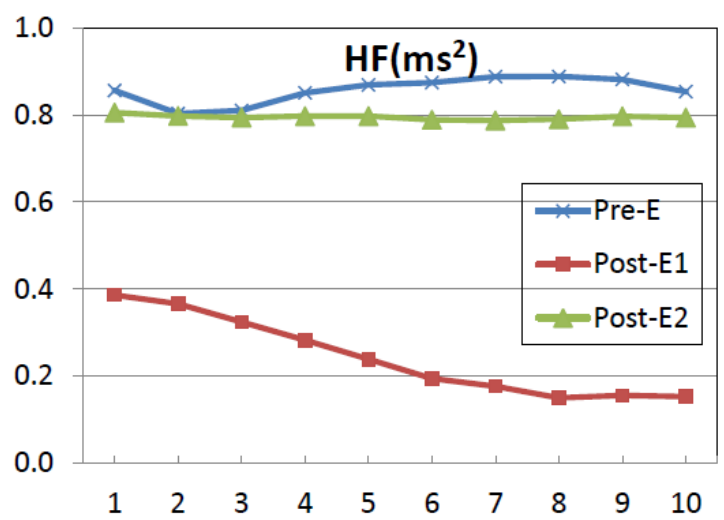

(b)

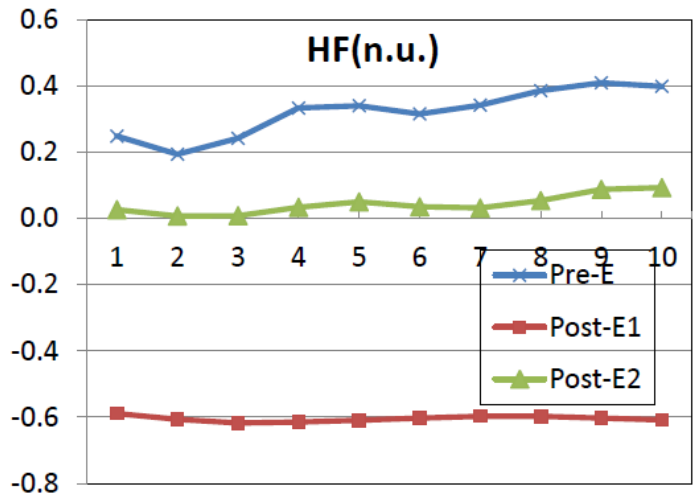

(d)

Fig. 2. The correlations between $\mathrm{SD} 2_{\mathrm{m}}$ and frequency domain indices: (a) $\mathrm{LF}\left(\mathrm{ms}^{2}\right)$, (b) $\mathrm{HF}\left(\mathrm{ms}^{2}\right)$, (c) LF(n.u.), (d) HF(n.u.), and (e) LF/HF. 


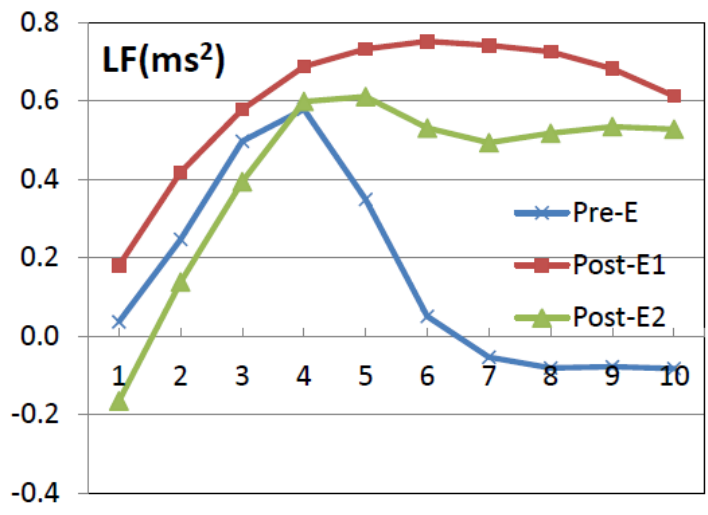

(a)

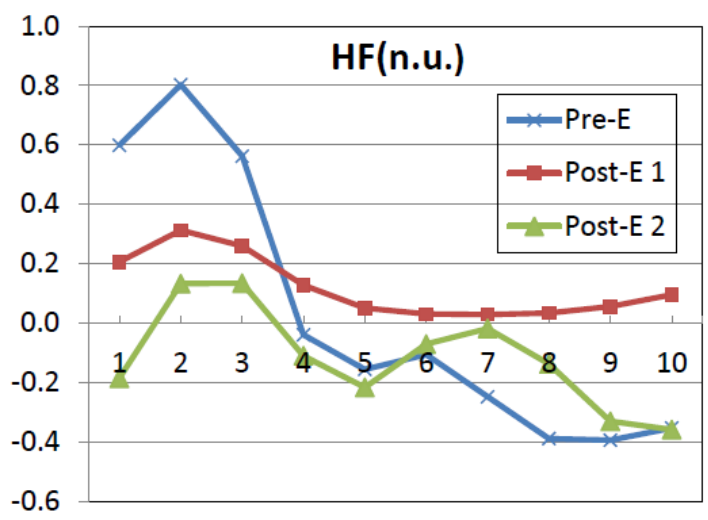

(c)

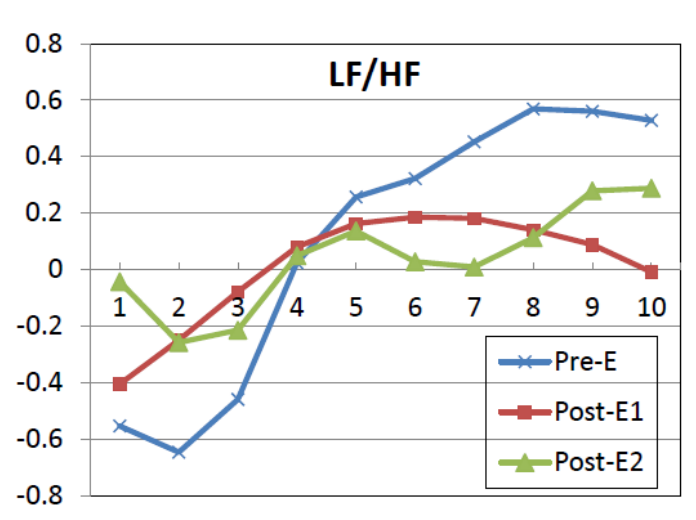

(e)

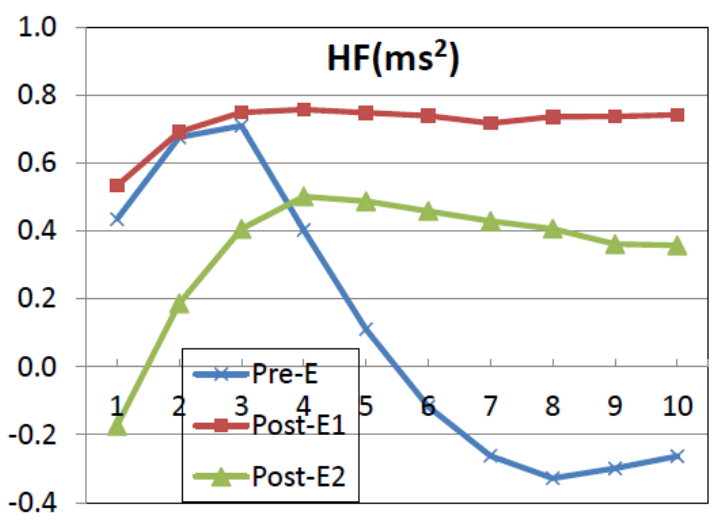

(b)

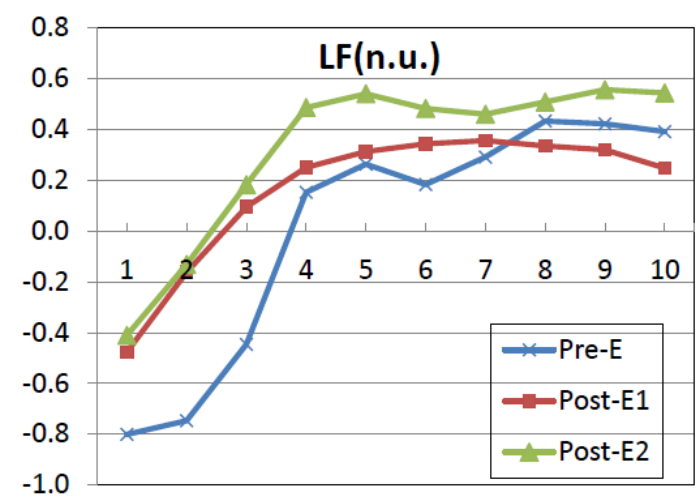

(d)

Fig. 3. The correlations between $\mathrm{SD} 1_{\mathrm{m}} / \mathrm{SD} 2_{\mathrm{m}}$ and frequency domain indices: (a) $\mathrm{LF}\left(\mathrm{ms}^{2}\right)$, (b) HF(ms ${ }^{2}$ ), (c) LF(n.u.), (d) HF(n.u.), and (e) LF/HF. 